rentée à la «pêche à la ligne ». Il faut d'abord comprendre la réaction et le mécanisme chimique qui la soustend. On peut ensuite concevoir un système capable de s'ajuster en trois dimensions aux réactifs, ainsi que la manière d'accélérer la réaction. Le dessin rationnel de molécules qui fonctionnent de façon prédictible est une nouveauté capitale en chimie.

\section{J.C.D.}

\footnotetext{
l. Waldrop MM. «Chemzymes» mimic biology in miniature. Science $1989 ; 248: 354-5$. 2. Corey EJ, Bakshi RK, ShibataS. Highly enantioselective borane reduction of ketones catalyzed by chiral oxazaborolidines. $\mathrm{J} \mathrm{Am}$ Chem Soc 1987; $109: 5551-3$.

3. Corey EJ, Bakshi RK, Shibata S, Chen CP, Singh VK. A stable and easily prepared catalyst for the enantioselective reduction of ketones. Applications to multistep syntheses. $J$ Am Chem Soc 1987 ; 109 : 7925-6.

4. Corey EJ, Jardine DS, Moleri T. Enantioselective route to a key intermediate in the total synthesis of forskoline. Tetrahedron Letters $1988 ; 29: 6409-12$.
}

\section{口I BRĖVES}

Gestations prolongées chez certains mammifères. La durée de gestation d'un édenté américain, le tatou (Dasypus novemcinctus) est de huit à neuf mois, y compris une période de trois à quatre mois avant l'implantation du blastocyste. Chez plusieurs femelles capturées au moment habituel de l'implantation (novembre à février), on a observé un délai supplémentaire d'un an, attribué au stress de la capture; dans deux cas, la gestation a duré plus de 30 mois. La question se pose de savoir si ces diapauses prolongées n'existent que chez le tatou, et les auteurs [1] suggèrent d'en faire une recherche systématique dans des espèces qui présentent normalement une diapause, telles que le blaireau européen (Meles meles, dix mois) ou le glouton (ou carcajou, Gulo gulo, six à huit mois).

[1. Storrs EE, Burchfield HP. Nature $1989 ; 340$ : 106.]

$\mathrm{m} / \mathrm{s} n^{\circ} 8$ rol. 5. octobre 89

\title{
L'archéologie moléculaire
}

L'évolution moléculaire s'étudie en comparant des protéines ou des acides nucléiques dans des espèces plus ou moins proches, ou dans des races différentes d'une même espèce. Un puissant stimulant lui viendrait de la connaissance des espèces disparues, mais dont des traces analysables subsisteraient, ou d'individus appartenant à une espèce encore présente, ayant vécu dans une période reculée, chez l'homme en particulier. Les premières méthodes dont on ait disposé concernaient les protéines. On sait que certaines liaisons peptidiques restent stables des millions d'années, et que la survie de ribosomes et de chromatine a pu être mise en évidence chez des insectes tirés d'ambre vieux de 40 millions d'années [1]. Deux difficultés ont entravé les progrès dans ce domaine : les protéines les plus accessibles, par exemple le collagène de l'os, ont une structure très uniforme à travers les espèces; surtout les protéines qui sont solubles ont subi de telles modifications post-mortem que les résultats sont souvent trompeurs. Cependant quelques études immunologiques ont pu être menées à bien : la plus connue porte sur le muscle de mammouth sibérien, resté congelé pendant 40000 ans ; elle a montré la parenté immunologique de ses protéines avec celles des éléphants d'Asie et d'Afrique. En génétique des populations, les systèmes $\mathrm{ABO}$ et HLA ont fourni et pourraient encore fournir des renseignements intéressants.

C'est naturellement l'étude de l'ADN qui suscite le plus d'espoirs depuis qu'on a montré vers 1980 qu'il peut être retrouvé dans certains tissus anciens. Une équipe californienne s'est attaquée à ce problème [2]. Les principales difficultés à surmonter sont la dégradation de l'ADN (la taille des fragments ne dépasse pas quelques centaines de bases) et les altérations diverses qui portent sur sa structure. Elles rendent le clonage très difficile sinon impossible car les molécules intactes sont noyées dans la masse de celles qui sont endommagées. Là comme ailleurs, la découverte de la méthode d'amplification de l'ADN dite PCR permet des progrès décisifs. On possède avec elle un outil idéal pour amplifier un petit nombre de séquences intactes au sein d'un vaste excès d'ADN altéré. En effet, au cours de la réaction, la plupart des molécules endommagées ne se répliquent pas du tout du fait de liaisons intra- ou intermoléculaires. Si elles se répliquent, le processus sera ralenti par des lésions telles que des sites ayant perdu leur base, et l'amplification des séquences intactes prendra le dessus. On ne dépasse guère, toutefois, une longueur de 150 bases, et on observe une forte corrélation inverse entre l'efficacité de l'amplification et la taille du fragment obtenu. Enfin, avant de conclure que ce qu'on a analysé est bien de l'ADN ancien, il faut multiplier les précautions pour éviter, ou reconnaître, une contamination par de l'ADN moderne. Curieusement, on ne trouve pas de corrélation entre les altérations de l'ADN et l'ancienneté du spécimen. Les deux facteurs essentiels sont: d'abord la vitesse de dessiccation, qui protège contre l'hydroxylation; puis, une fois les tissus secs, la protection plus ou moins bonne contre l'oxydation. Le rendement ne dépasse pas $1 \%$ de ce que l'on obtient avec de l'ADN récent. Les problèmes que l'on étudie sont différents selon qu'on s'adresse à des espèces éteintes ou toujours présentes, de l'ADN humain ou non humain, de l'ADN nucléaire ou mitochondrial. Ce dernier présente deux avantages : il est présent en de nombreuses copies par cellule; les chances sont bonnes pour que quelques molécules soient intactes. En outre, son taux de mutation est 
beaucoup plus élevé, et c'est un avantage car sur les périodes qu'on peut suivre, quelques milliers ou dizaines de milliers d'années, il a pu avoir le temps d'évoluer, alors qu'un gène nucléaire n'aura pas été modifié de façon appréciable.

A quels problèmes cette discipline, encore à ses débuts, a-t-elle déjà pu s'attaquer? On a surtout examiné des problèmes de classification d'espèces et d'archéologie humaine.

1. Problèmes taxonomiques. Ils consistent surtout à compléter les relations entre les espèces, en insérant dans les arbres phylogéniques des espèces aujourd'hui disparues. Si l'on n'est pas surpris de voir confirmée la parenté entre mammouth et éléphant, on a pu rapprocher, à l'intérieur du genre equus, le couagga (ou quagga), animal disparu depuis 150 ans, du zèbre plutôt que du cheval, grâce à l'ADN extrait d'un specimen de musée [2].

2. Chez l'homme, on s'est surtout adressé aux momies, c'est donc de l'Égypte que sont tirés la plupart des tissus analysés, comprenant des échantillons datant de 2000 à 5000 ans. Des renseignements détail- lés ont été obtenus à partir du foie de Nekht-ankh, un prêtre de haut rang qui vivait il y a 4000 ans. En particulier, une séquence d'ADN mitochondrial d'environ 50 paires de bases comportait deux différences avec la séquence de référence [3]. Une autre séquence d'ADN mitochondrial a été obtenue par le même auteur [4] à partir du cerveau d'un enfant ayant vécu il y a 7000 ans en Floride; cette séquence appartient à une lignée d'ADNmt qui, actuellement, est rarement trouvée en Europe et jamais en Amérique. Enfin, sur une autre momie, celle d'un enfant de un an datant de 2400 ans, le même Pääbo a pu cloner un $\mathrm{ADN}$ nucléaire de $3,4 \mathrm{~kb}$, contenant des séquences Alu [5].

Que peut-on attendre de l'archéologie moléculaire dans l'avenir? Outre les relations avec des espèces éteintes, on envisage essentiellement des processus de génétique moléculaire qui soient rapides, c'est-à-dire susceptibles de se dérouler dans un délai de $10^{4}$ années. Ils incluent les variations de l'ADNmt, mais aussi des recombinaisons dans des séquences de minisatellites, dont la fréquence de mutation apparente est de l'ordre de $10^{-2}$ par locus et par génération [6]. Les égyptologues pourront se délecter à tenter d'établir les relations entre les dynasties de pharaons, et même entre les membres de chacune de ces familles. Enfin leur évolution rapide permettra l'étude moléculaire de parasites, de virus et de nombreuses plantes domestiques.

J. C. D.

1. Poinar GO Jr, Hess R. Ultrastructure of 40 million year old insect tissue. Science $1982 ; 215: 1241.2$.

2. PääboS, HiguchiRG, Wilson AC. Ancient DNA and the polymerase chain reaction. The emerging field of molecular archaeology. J Biol Chem 1989; 264 : 9709-12. 3. Pääbo S. Ancient DNA : extraction, characterization, molecular cloning and enzymatic amplification. Proc Natl Acad Sci USA $1989 ; 86: 1939-43$.

4. Pääbo S, Gifford JA, Wilson AC. Mitochondrial DNA sequences from a 7000-year old brain. Nucleic Acids Res 1988 ; 16 : 9775 . 87.

5. Pääbo S. Molecular cloning of ancient egyptian mummy DNA. Nature $1985 ; 314$ : 644-5.

6. Jeffreys AJ, Royle NJ, Wilson V, Wong Z. Spontaneous mutation rates to new length alleles at tandem-repetitive hypervariable loci in human DNA. Nature 1988; $332: 278-81$.

\section{口 BRÈVES}

Les effets vasodilatateurs de la vasopressine. On a longtemps considéré que la vasopressine avait d'une part des effets antidiurétiques (qui passent par des récepteurs $V_{2}$ dont l'activation accroît l'AMP cyclique intracellulaire), d'autre part des effets vasoconstricteurs, entraînant une élévation de la pression artérielle, dépendant de l'activation de récepteurs $V_{1}$ qui conduit à l'augmentation de la concentration intracellulaire de calcium. L'étude de Hirsch et al. [2] démontre que la vasopressine administrée par voie intra-artérielle entraîne chez l'homme normal une augmentation du débit sanguin (musculaire) de l'avant-bras perfusé (mais non de l'avant-bras controlaté- antagoniste $V_{1}$ potentialise l'effet vasodilatateur de la vasopressine. La perfusion intra-artérielle d'un agoniste $V_{2}$ produit également une élévation du débit sanguin. La vasopressine entraîne donc un effet direct vasodilatateur qui dépendrait de l'activation de récepteurs $V_{2}$ vasculaires. En revanche, le débit sanguin digital est diminué par la vasopressine : les effets de celle-ci varient donc selon les territoires vasculaires, dépendant probablement de l'équilibre local entre les récepteurs $V_{1}$ et $V_{2}$. Les résultats de Suzuki et al. [3] vont dans le même sens et apportent des informations complémentaires. La vasopressine exerce un effet biphasique sur la circulation de l'avant-bras: vasoconstricteur à faibles doses, vaso- dilatateur à fortes doses. Celui-ci ne dépend pas de la libération de prostaglandines puisqu'il n'est pas bloqué par l'indométhacine. En revanche, il est prévenu par l'administration de $\mathrm{CaCl}_{2}$ (qui atténue la vasodilatation induite par le GMP cyclique). Il est possible que l'effet vasodilatateur de la vasopressine soit lié au GMP cyclique par un mécanisme encore inconnu.

[1. Bichet DG, et al. $N$ Engl J Med $1988 ; 318: 881-7$.

[2. Hirsch AT, et al. J Clin Invest 1989 ; 84 : 418-26.]

[3. Suzuki S, et al. J Clin Invest $1989 ; 84: 427-34$.] 
Recette pour construire un système visuel dans une boîte de Pétri. Vous prenez deux explants chez un fotus de rat de 16 jours - un thalamus visuel qui vient de se mettre en place et un cortex cérébral visuel qui est encore dans les langes - et vous les placez côte à côte dans le verre. Laisser pousser deux à trois semaines et... c'est tout [1]. Le cortex explanté se développe en formant les six couches de neurones bien différenciés qu'on lui connaît in vivo. Les neurones du thalamus visuel font pousser des axones qui passent au travers de l'explant cortical pour rejoindre les neurones de la couche numéro IV qu'ils contactent. A l'inverse, les neurones corticaux de la couche VI contactent les neurones de l'explant thalamique, comme s'ils se trouvaient encore dans l'animal en développement. Et l'enregistrement électrophysiologique démontre que tous ces contacts sont fonctionnels. La prochaine étape est-elle l'introduction d'un troisième explant, une rétine par exemple, avec activation du circuit par une stimulation lumineuse?

[1. Yamamoto N, et al. Science 1989 ; $245: 192-4$.

Clonage moléculaire d'ADN complémentaires codant pour le récepteur des hormones LH (luteinizing hormone) et gonadotrophine chorionique. L'ADNc du récepteur de rat a été cloné par l'équipe new yorkaise de P.H. Seeburg [1] alors que celui du récepteur porcin a été cloné par les équipes INRA de J. Garnier (Jouyen-Josas, France) et INSERM d'E. Milgrom (Kremlin-Bicêtre, France). Les protéines, de 700 [1] et 696 [2] acides aminés, possèdent un domaine d'un peu plus de 300 résidus contenant sept segments transmembranaires et appartiennent donc à la même famille de récepteurs que ceux de l'acétylcholine (récepteur muscarinique), que le récepteur $\beta_{2}$ adrénergique ou la rhodopsine. Contrairement à eux, cependant, le récepteur de la LH-gonadotrophine chorionique a également un très $\mathrm{m} / \mathrm{s} n^{\circ} 8 \mathrm{vol} .5$, oclobre 89 grand domaine extracellulaire (environ 340 acides aminés) qui est un probable site de liaison des hormones. Plusieurs messagers sont détectés dans les ovaires, dont une forme prédominante de 4,4 ou 4,7 kb, et des formes minoritaires dont certaines semblent coder pour des molécules tronquées de récepteur, dépourvues du domaine intramembranaire. Probablement engendrées par épissage alternatif, la signification de ces espèces minoritaires est peu claire [2]. Le récepteur synthétisé sous le contrôle de l'ADNc cloné est fonctionnel. Des cellules l'exprimant après transfection répondent à la stimulation hormonale par une activation de l'adénylate cyclase [1].

[1. McFarland KC, et al. Nature $1989 ; 245$ : 494-9.]

[2. Loosfelt $\mathrm{H}$, et al. Nature 1989 ; 245 : 525-8.]

$\square$ Le nerve growth factor (NGF) serait-il à l'origine de certaines épilepsies, c'est l'hypothèse formulée par Gall et Isackson [1]. Ils ont en effet remarqué que des crises limbiques induites expérimentalement chez le rat provoquent une augmentation majeure et transitoire des messagers de la sous-unité $\beta$ du NGF dans certaines régions du cerveau. $\mathrm{Ce}$ phénomène a en particulier lieu dans les neurones du gyrus dentatus de l'hippocampe, dont le rôle dans les crises limbiques est connu. Lors de telles crises, un grand nombre de gènes est anormalement exprimé. L'intérêt porté au NGF tient essentiellement à la capacité qu'a ce facteur trophique de provoquer des altérations structurales importantes dans les neurones cibles, favorisant en particulier leur excitabilité. L'hyperproduction de NGF pourrait donc provoquer l'hypersensibilité de certaines populations neuronales à des activations qui, dans des conditions normales, auraient des effets infraliminaires. Il ne s'agit certes que d'une hypothèse de travail, mais il est pro- bable qu'clle ne le restera pas longtemps, étant donné les possibilités thérapeutiques qu'ouvrirait sa confirmation.

[1. Gall CM, Isackson PJ. Science $1989 ; 245$ : 758-61.]

Excès de mutations somatiques dans le syndrome de Werner. Le syndrome de Werner est une maladie récessive autosomique entraînant un vieillissement accéléré, in vivo comme in vitro. Sur des fibroblastes en culture, on a mis en évidence une réponse mitogénique très basse à certains facteurs de croissance tels que le PDGF ( $m / s n^{\circ} 4$, vol. 3, p. 244). En raison des difficultés du travail avec des fibroblastes primaires, qui ne se répliquent qu'un très petit nombre de fois, une équipe de Seattle (WA, USA) a utilisé des fibroblastes immortalisés par le SV40[1]. Elle a recherché le taux de mutations comparé de deux cultures de syndrome de Werner et de deux témoins, en prenant l'exemple commode de l'enzyme HGPRT. Lorsqu'on les cultive sur un milieu contenant de la 6thioguanine, les cellules normales ne peuvent survivre et seules poussent celles dans lesquelles une mutation a inactivé le gène de HGPRT. Les auteurs ont fait les constatations suivantes: (a) le taux de mutation est dix fois plus élevé dans les cellules Werner que dans les témoins; (b) parmi les mutants, la proportion de délétions, partielles ou complètes, du gène HGPRT, est beaucoup plus élevée dans le Werner que chez les témoins. Ces délétions importantes (plus de la moitié d'entre elles dépassent $20 \mathrm{~kb}$ ) caractérisent pour les auteurs le syndrome de Werner comme un "phénotype mutateur", qui pourrait jouer un rôle importan t dans la pathogénie du syndrome de Werner et peut-être aussi d'autres processus du vieillissement. Une restriction dans l'interprétation tient au fait que, dans le Werner, les rares cellules qui survivent assez pour s'immortaliser ne sont peut-être pas parfaitement représentatives.

[1. Fukuchi KI, et al. Proc Natl Acad Sci USA 1989; 86 : 5893-7.]
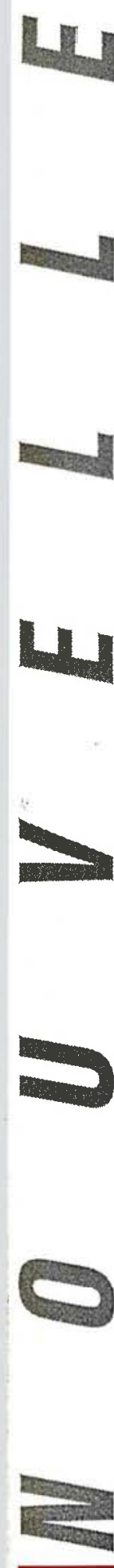